\title{
IL PORTO DI GENOVA E LE SCULTURE ANTELAMICHE DI FIDENZA - RICONSIDERAZIONE DELLA QUESTIONE PROVENZALE
}

\section{YOSHIE KOJIMA}

UDC: $711.453 .4(450.421) " 11 "$ $726.6(450.421+450.45) " 11 "$

Original scientific paper

Manuscript received: 21. 02. 2016.

Revised manuscript accepted: 24. 04. 2016.

DOI: 10.1484/J.HAM.5.111351
Y. Kojima

Sophia University—Faculty of Humanities

Kioi-cho 7-1, Chiyoda-ku Tokyo 102-8554, Japan

Since the end of the nineteenth century, scholars, especially Germans, ascribed to Provençal art the origin of the monumental sculpture of Benedetto Antelami, and in particular, that of the Cathedral of Fidenza. This thesis provoked a fervent debate, especially with regard to a hypothetical trip to Provence Antelami. Here, I would first like to review the noteworthy history of the studies on the sculpture of Antelami. Then, I will re-examine this problem, which still remains an issue, by focusing on the role of the port of Genoa. Indeed, in Genova the 'magistri antelami' in which Benedetto Antelami belonged have been active since at least the beginning of the twelfth century. Interestingly, Genova at that time enjoyed the status of a powerful maritime republic and had a strong commercial penetration in Provence. Regarding the relationship between Genoa and Fidenza, the role of the Pallavicino family should have been relevant.

Keywords: Benedetto Antelami, magistri antelami, Cathedral of Fidenza, Cattedrale of Genova, Porta Soprana in Genova, Chiostro Sant'Andrea della Porta in Genova, Baptistery of Parma, Saint-Gilles-du-Gard, Saint-Trophime in Arles, Pallavicino family

Già dalla fine dell'Ottocento gli studiosi, soprattutto tedeschi, ipotizzavano un'origine provenzale della scultura monumentale antelamica, soprattutto quella dell'attuale Cattedrale di Fidenza, già chiesa collegiata di Borgo San Donnino (figg. 1, 2) ${ }^{1}$. Questa tesi suscitò un fervente dibattito, in particolare per quanto riguarda un ipotetico viaggio in Provenza di Benedetto Antelami o anche dei suoi seguaci. In questa sede vorrei prima parlare dell'insolita "fortuna" della scultura antelamica, e poi riesaminare questa problematica, che rimane aperta ancora oggi, mettendo a fuoco in particolare il ruolo del porto di Genova. A Genova, infatti, furono attivi almeno dal XII secolo i "magistri antelami” ai quali Benedetto Antelami apparteneva, e, come è ben noto, nella Cattedrale genovese ci sono leoni stilofori in stile antelamico (figg. 3, 4) ${ }^{2}$.
Ma prima vorrei soffermarmi su alcune indubbie concordanze stilistiche tra l'arte provenzale e quella antelamica a Fidenza. Gli elementi naturalistici e classicheggianti osservabili nei Profeti nella facciata del Duomo di Fidenza concordano con quelli presenti nella scultura provenzale, specie della facciata della chiesa abbaziale di Saint-Gillesdu-Gard e della facciata e della galleria nord del chiostro di Saint-Trophime ad Arles (figg. 5-9). Ė alquanto evidente anche l'affinità dei motivi decorativi di forte carattere antico (figg. 10-14). E come mostrato chiaramente già nel 1922 da Richard Hamann, gli Angeli che guidano due famiglie nella facciata di Fidenza richiamano fortemente un Angelo di Saint-Gilles (figg. 15,16)3.

Tra la fine del XIX secolo e l'inizio del XX, ferveva la discussione sull'importanza dell'arte provenzale per la ri-

\footnotetext{
${ }^{1}$ Sulla storia e la Cattedrale di Fidenza vedi G. Laurini, San Donnino e la sua città. Memorie storiche, Borgo San Donnino, 1924; G. Laurini, S. Donnino e la sua chiesa, Fidenza, 1927.G. de Francovich, Benedetto Antelami. Architetto e scultore e l'arte del suo tempo, 2voll, Milano-Firenze, 1952; A. C. Quintavalle, Romanico padano, civiltà d'Occidente, Firenza, 1969; R. Tassi, Il Duomo di Fidenza, Parma 1973; A. Aimi, Storia di Fidenza, Parma, 2003. A. Aimi, A. Copelli, Storia di Fidenza, Parma, 1982; A. C. Quintavalle, Benedetto Antelami, catalogo della mostra (Parma, Salone delle Scuderie in Pilotta, 31 marzo-30 settembre 1990), Parma, 1990; M. Woelk, Benedetto Antelami. Die Werke in Parma und Fidenza, Münster, 1995, W. Sauerländer, Benedetto Antelami: per un bilancio critico, in Benedetto Antelami e il Battistero di Parma, ed. C. Frugoni, Torino, 1995, pp. 3-69; Idem, Fidenza e la sua cattedrale, in Medioevo europeo di Jacques le Goff, catalogo della mostra (Parma, 28 settembre 2003-6, gennaio 2004), a cura di D. Romagnoli, Milano, 2003., pp. 231-238; Y. Kojima, Storia di una cattedrale. Il Duomo di San Donnino a Fidenza: il cantiere medievale, le trasformazioni, i restauri, Pisa, 2006; G. Freni, Space, images, and the public at the duomo of Fidenza, in Meaning in Motion, ed. N. Zcholmelidse-G. Freni, Princeton N.J., 2011, pp. 95-130; Y. Kojima, Iconografia per Sacrum Imperium: rilievi nella facciata del Duomo di San Donnino a Fidenza, in Conosco un ottimo storico dell'arte...per Enrico Castelnuovo, ed. M. Donato, M. Ferretti, Pisa, 2012, pp. 77-82.

${ }^{2}$ Sui leoni stilofori nella facciata della cattedrale di Genova vedi C. Di Fabio, Il cantiere duecentesco della Cattedrale, in Niveo de marmore: l'uso artistico del marmo di Carrara dall' XI al XV secolo, catalogo della mostra (Sarzana, 1 marzo-3 maggio 1992), ed. E. Castelnuovo, Genova, 1992, pp. 181-189; F. Cervini, Il portali della Cattedrale di Genova e il Gotico europeo, Firenze, 1993; C. Di Fabio, "Opus francigenum" dans une ville méditerranéenne: la cathédrale de Gênes dans la premier XIII e siècle, in Histoire médiévale et archéologie 11, 2000, pp. 246-276; A. R. Calderoni Masetti, Benedetto Antelami fra Genova e Parma, in Medioevo: le officine, atti del convegno internazionale di studi (Parma, 22-27 settembre 2009), ed. A. Q. Quintavalle, Milano, 2010, pp. 302-311; L. Cavazzini, Un restauro cinquecentesco per i leoni antelamici del Duomo di Genova, in Forme e Storia. Scritti di arte medievale e moderna per Francesco Gandolfo, ed. W. Angelelli, F. Pomarici, Roma, 2011, pp. 239-246; C. Di Fabio, La Cattedrale di Genova fra 1200 e 123o: esiti romanici, cantiere gotico, progetto architettonico e obiettivo di comunicazione, in La Cattedrale di San Lorenzo a Genova, ed. A. R. Calderoni Masetti-G. Wolf, Modena, 2012, pp. 59-74. Laura Cavazzini ha individuato nella testa del leone stiloforo di sinistra l'esito di una rilavorazione cinquecentesca.

${ }^{3}$ R. Hamann, Deutsche und Französische Kunst im Mittelalter, 1, Südfranzösische Protorenaissance und ihre Ausbreitung in Deutschland auf dem Wege durch Italien und die Schweiz, Marburg, 1922, p. 72.
} 


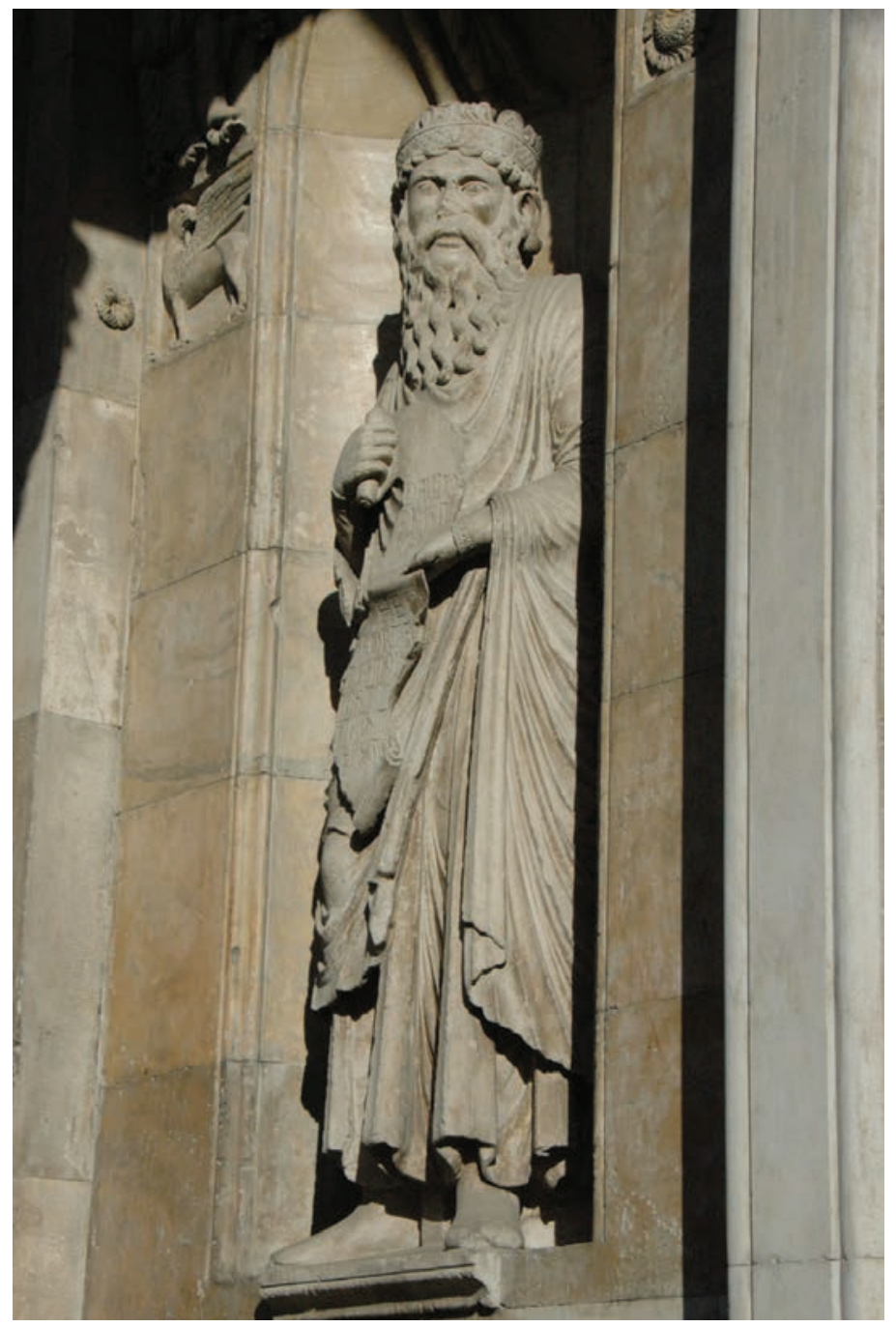

Fig. 1. Cattedrale di Fidenza, facciata, nicchia settentrionale, Davide

nascita della scultura monumentale in Emilia ed in Francia settentrionale. Fu Wilhelm Vöge che iniziò questo dibattito nella sua celebre monografia intitolata Die Anfänge des monumentalen Stiles im Mittelalter del 1894 che ipotizzava l'origine della scultura monumentale romanica nel Sud della Francia, da cui dipenderebbe quella gotica, poi sviluppatasi

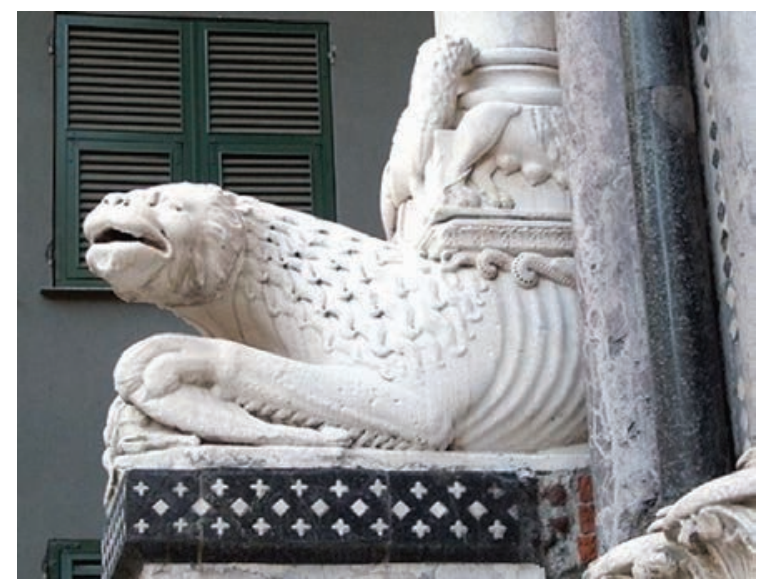

Fig. 3. Cattedrale di Genova, facciata, Leone stilifolo di sinistra

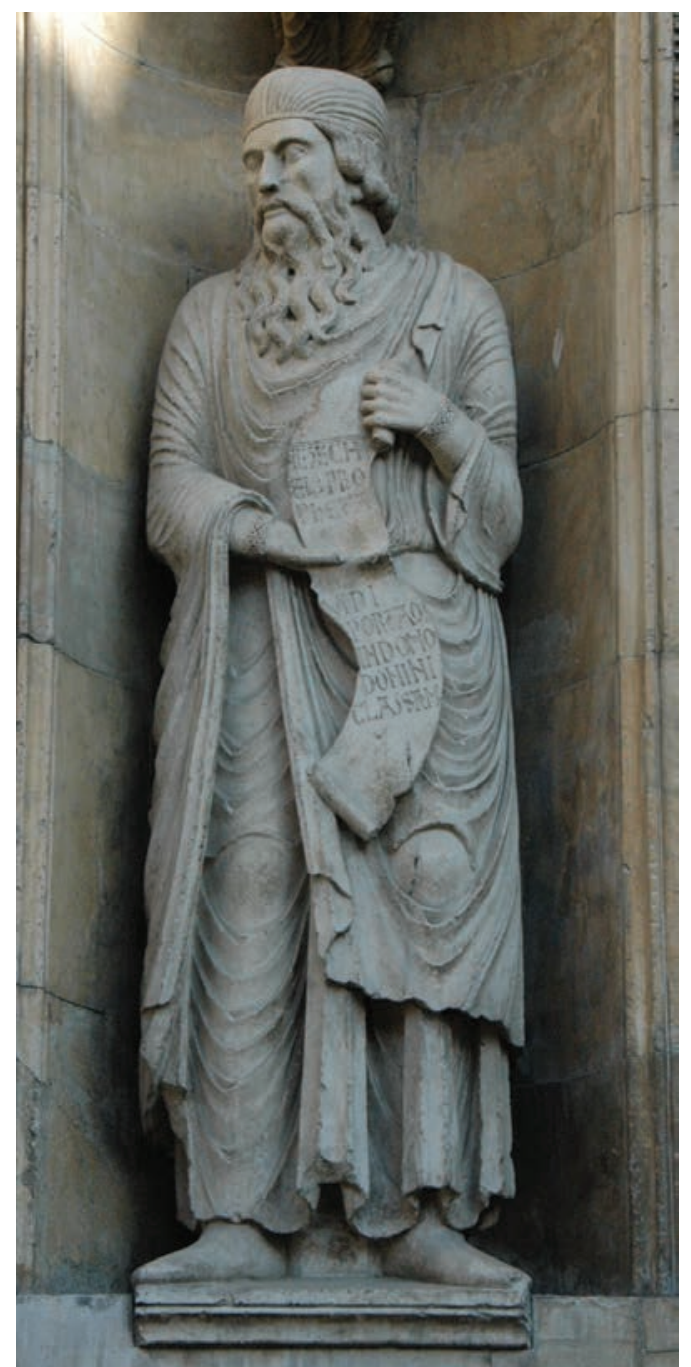

Fig. 2. Cattedrale di Fidenza, facciata, nicchia meridionale, Ezechiele nell'Ile-de-France ${ }^{4}$. Facendo riferimento a questo studio, Max Georg Zimmermann nel 1897 ha suggerito rapporti formali ed iconografici delle sculture antelamiche con la scultura protogotica dell'Ile-de-France e soprattutto con quella romanica provenzale di Saint-Trophime ad Arles e di Saint-Gilles 5 . Con questa tesi, che inaugura la fortuna

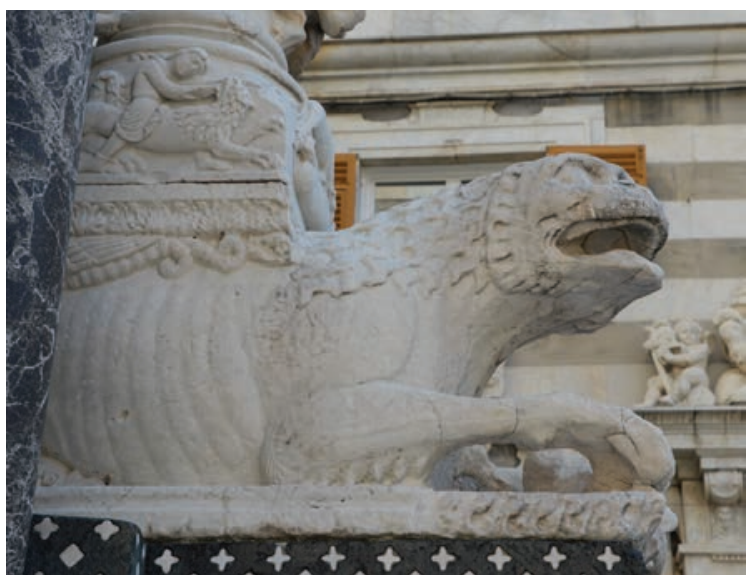

Fig. 4. Cattedrale di Genova, facciata, Leone stilifolo di destra

\footnotetext{
${ }^{4}$ W. Vöge, Die Anfänge des monumentalen Stiles im Mittelalters, Strassburg, 1894. Cf. A. Peroni, Echi di Wilhelm Vöge nella storiografia artistica italiana a proposito della 'questione provenzale', in Arte medievale N.S.5-2, 2007, pp. 65-85; W. Sauerländer, "Die Anfänge des monumentalen Stiles", nach dem Ende der Stilgeschichte, in Mittelalterliche Bauskulptur in Frankreich und Spanien, ed. C. Rückert, J. Staebel, Frankfurt am Main, 2010, pp. 23-47; Wilhelm Vöge und Frankreich, Akten des Kolloquiums aus Anlaß des 5o. Todestages von Wilhelm Vöge (Freibug, 2. Mai 2003) ed. W Schlink, Freiburg, 2014.

${ }^{5}$ M. G. Zimmermann, Oberitalische Plastik im frühen und hohen Mittelalter, Leipzig, 1897, pp. 98-157.
} 


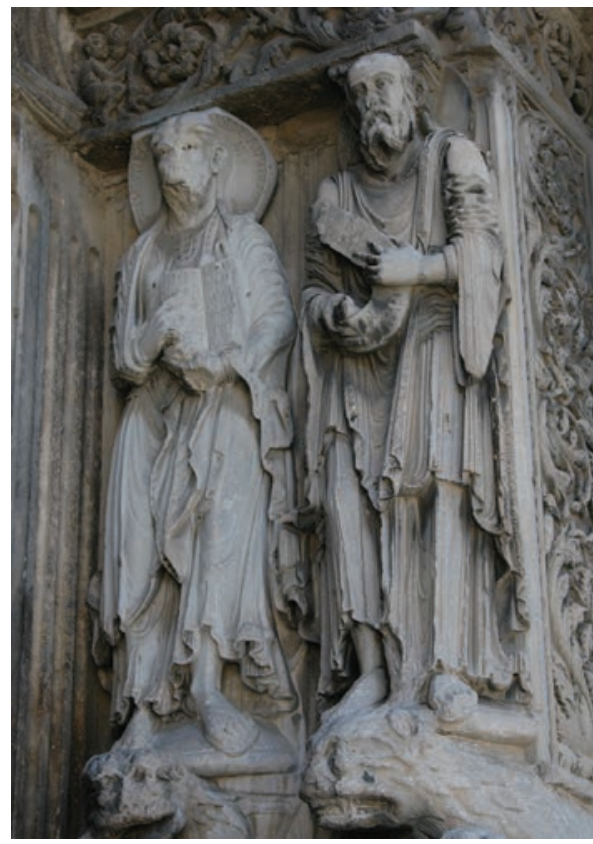

Fig. 5. Saint-Gilles-du-Gard, facciata, San Giacomo maggiore e San Paolo (destra)

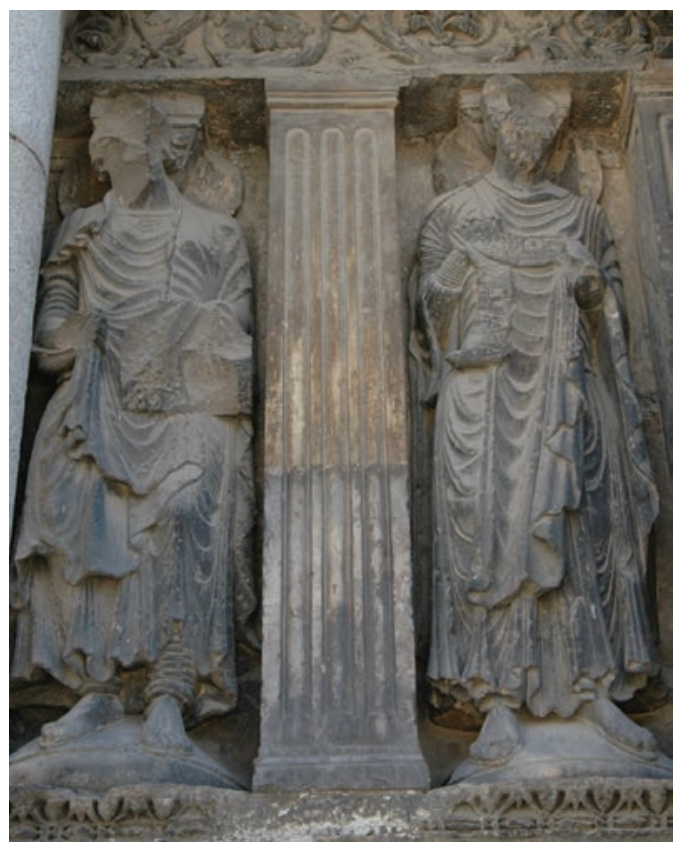

Fig. 6. Saint-Gilles-du-Gard, facciata, San Matteo e San Bartolomeo

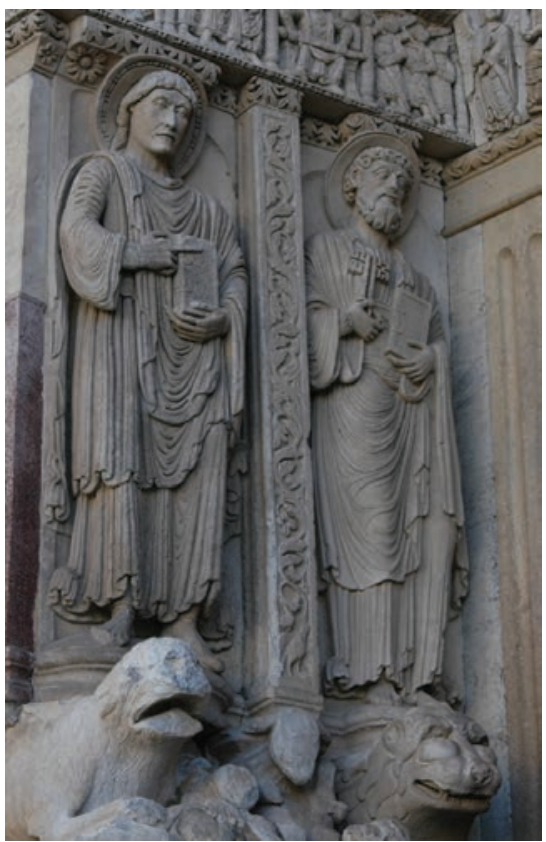

Fig. 7. Arles, Saint-Trophime, facciata, San Giovanni e San Pietro

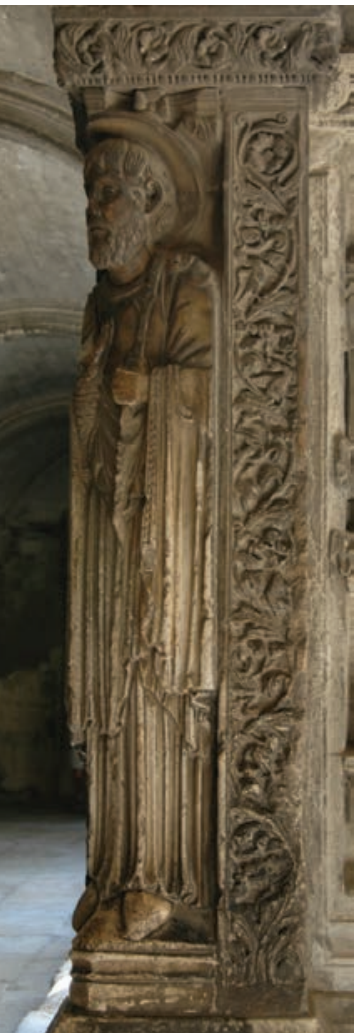

Fig. 9. Arles Saint-Trophime, chiostro, angolo delle gallerie Nord-Est, San Trofimo nel Battistero di Parma è stata ricavata riscolpendo una scultura romana raffigurante una figura virile togata (figg.

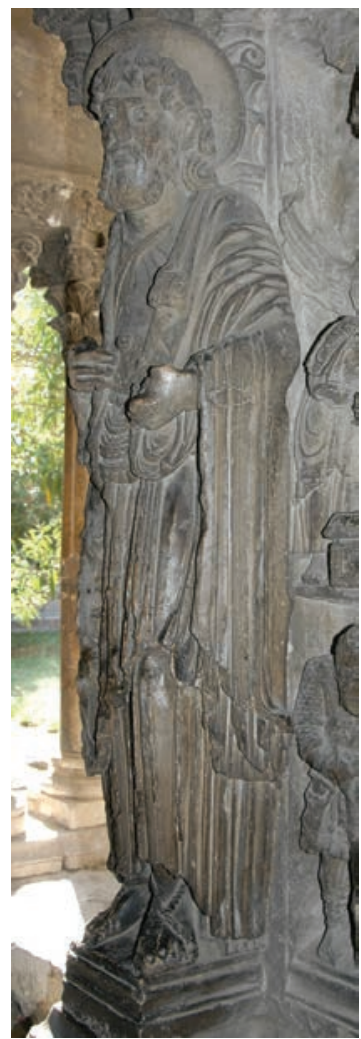

Fig. 8. Arles, Saint-Trophime, chiostro, angolo delle gallerie Nord-Ovest, San Pietro

novecentesca dell'artista, si approda al problema del rapporto dello stile antelamico con l'arte provenzale. Testo fondamentale per tale discussione è l'articolo pubblicato nel 1902 da Vöge col titolo Der Provençalische Einfluss in Italien und das Datum des Arler Porticus 6 . Lo studioso tedesco si spinse fino a supporre che Antelami potesse essere stato in Provenza, punto di partenza per l'ipotesi della formazione provenzale del maestro che è stata sostanzialmente accettata da noti studiosi, ad esempio Émile Bertaux, Georg Vitzhum e Wolfgang Volbach, Paul Frankl, Rene Jullian, George Crichton, e Geza de Francovich77. Gli studi di quest'ultimo, ancora oggi utili per le loro minuziose osservazioni, hanno influito largamente sulle ricerche successive che trattano il capporto tra larte provenzale e quella antelamica. D'altro canto, successivamente, in reazione alle ipotesi sui frequenti spostamenti degli scultori romanici, si è manifestato scetSauerländer e Xavier Barral i Altet ${ }^{8}$.

Se nelle sculture provenzali si può segnalare la derivazione diretta dalla statuaria antica, cioè dalle vestigia dei monumenti antichi della zona, anche a Fidenza è possibile ipotizzare un processo analogo. In effetti, anche nelle vicinanze di Fidenza, ad esempio a Veleia ${ }^{9}$, sono state ritrovate diverse statue antiche, e inoltre Fidenza stessa era un municipium romano ${ }^{10}$. Dato ancor più significativo, 


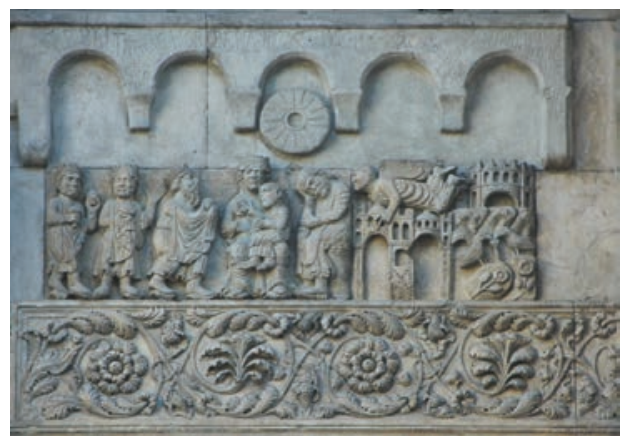

Fig. 10. Cattedrale di Fidenza, facciata, Adorazione dei Magi e Sogno di Giuseppe

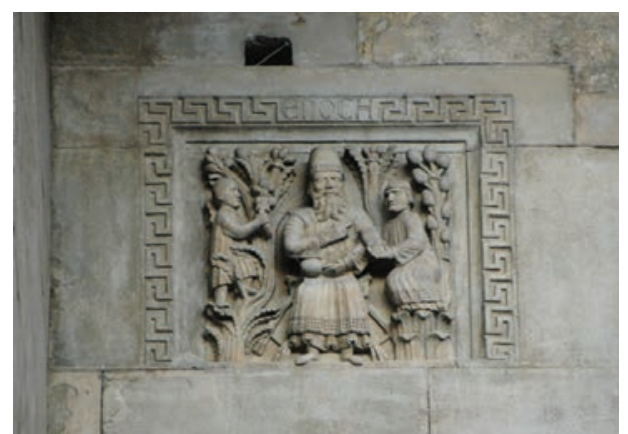

Fig. 11. Cattedrale di Fidenza, facciata, Enoch

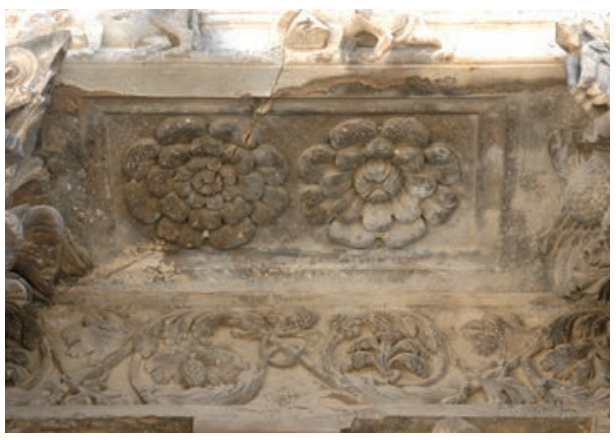

Fig. 12. Saint-Gilles-du-Gard, facciata

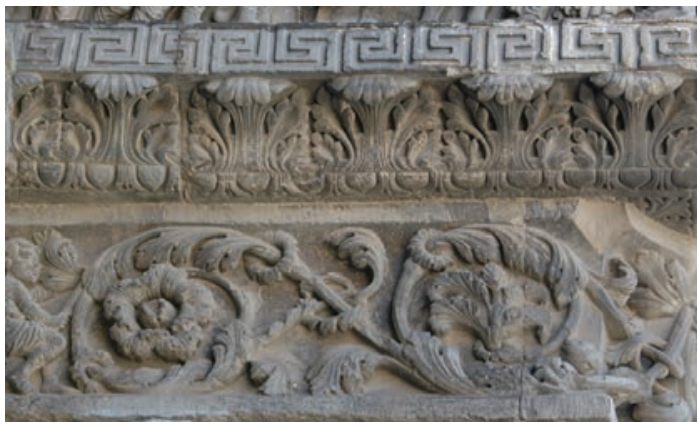

Fig. 13. Saint-Gilles-du-Gard, facciata

$17,18)^{11}$. Nell'Angelo, in particolare nei panneggi, è palese l'intento di imitare la statuaria antica. Inoltre, nei Profeti di Fidenza si possono segnalare caratteri divergenti da quelli

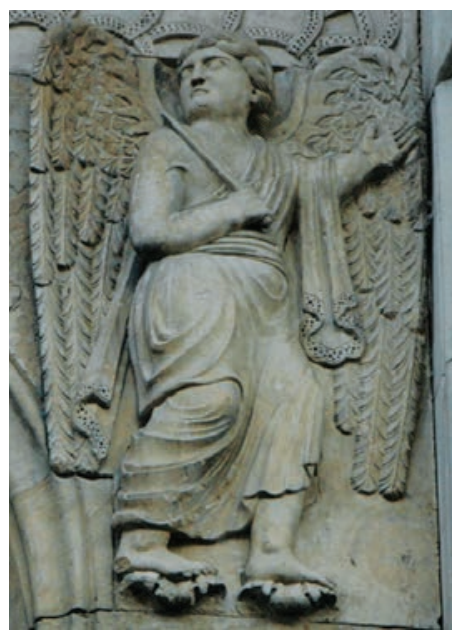

Fig. 15. Cattedrale di Fidenza, facciata, sopra la nicchia settentrionale, Angelo conducente una famiglia

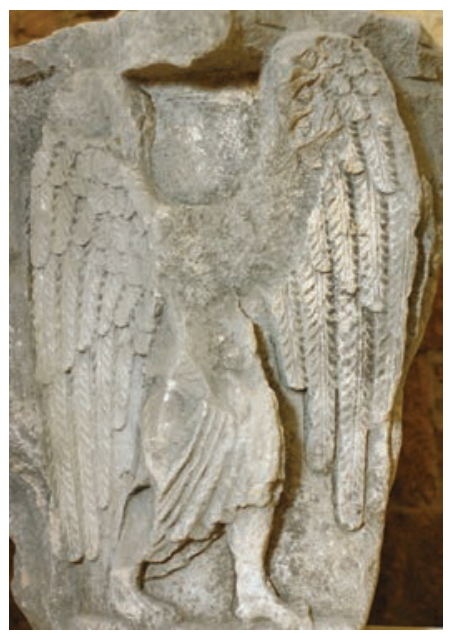

Fig. 16. Saint-Gilles-du-Gard, Musée de la Maison Romane, Angelo priveniente dall'abbazia

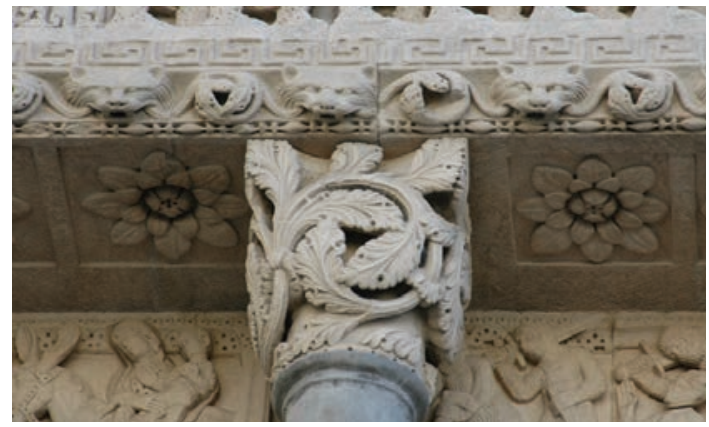

Fig. 14. Arles, Saint-Trophime, facciata

tipici della scultura romanica provenzale. A Fidenza non si riscontrano le espressioni molto marcate o quasi crudeli delle fisionomie delle opere provenzali. Anche le modalità di collocazione delle figure nella compagine architettonica della facciata, nelle nicche che affiancano il portale, diverge da quella provenzale, dove le sculture si allienano in una sorta di galleria (figg. 19-21). Oggi si ritiene che non si possa attribuire la diffusione dello stile solo a viaggi, peraltro ipotetici, di artisti. Appare opportuno discutere questa problematica in un più ampio contesto storico e politico. A questo proposito, in questa sede vorrei riflettere sul ruolo del porto di Genova.

Come è ben noto, a Genova è documentata dal XII secolo l'attività dei cosiddetti 'magistri antelami' provenienti dalle valli intorno ai laghi di Como e di Lugano, fra cui la Val d'Intelvi che ha dato loro il nome ${ }^{12}$. La loro competenza riguardava soprattutto l'edilizia, un settore in quell'epoca di grande sviluppo nel contesto della crescita demografica e economica della città' ${ }^{13}$.

È però scarsa la documentazione riguardo i loro interventi nelle fabbriche monumentali del XII secolo, come la chiesa di Santa Maria di Castello, il monastero di San Tom-

\footnotetext{
"P. Rockwell, La decorazione plastica: le tecniche d'intaglio, in Battistero di Parma, 2 voll., Parma, 1992, vol. 1, pp. 219-230; B. Zanardi, Relazione di restauro dei manufatti in pietra, policromi o meno, e delle superfici lapidee e in laterizio dell'interno e dell'esterno del Battistero di Parma, e alcune osservazioni di ordine materiale compiute durante i lavori, ibidem, pp. 249-268. Cf. anche V. Lassalle, Quelques cas d'influence de l'art antique dans l'art roman du Midi de la France et de l'Italie septentrionnale, in Les Cahiers de Saint-Michel de Cuxa 6, 1975, pp. 121-127; A. Hartmann-Virnich, L'image de l'art monumental antique dans l'architecture romane provençale, in Les Cahiers de Saint-Michel de Cuxa 39, 2008, pp. 47-64.

${ }^{12}$ Sui 'magistri antelami' lo studio tuttora fondamentale è quello di Gian Piero Bognetti, I magistri antelami e la Valle d'Intelvi (sec. XII), in Periodico storico comense 32, 1938, pp. 17-72. Vedi anche O. A. Ariatta, Die Antelami-Meister und das Intelvital, in Ostbairische Grenzmarken 11, 1969, pp. 112-116; E. Poleggi, Città e mastri antelami, una storia sequestrata, in Magistri d'Europa, ed. S. Della Torre et al., Milano, 1996, pp. 389-406; S. Lomartire, Magistri Campionesi a Bergamo nel Medioevo, da Santa Maria Maggiore al Battistero, in Arte \& storia 10, 2009, pp. 54-83; Idem, Comacini, Campionesi, Antelami, "Lombardi": problemi terminologici e storiografici, in Els "comacini" i l'arquitectura romànica a Catalunya, atti del convegno (Girona, 25-26 novembre 2005), ed. P. F. i Camps, J. C. i Sòria, Girona, 2010, pp. 9-31; M. Lazzati, Il significato del termine "Antelami" nei documenti medievali e nell'iscrizione di Benedetto Antelami nel Duomo di Parma, in Archivio storico ticinese 150, 2011, pp. 298-310.

${ }^{13}$ Riguardo l'attività dei 'magistri antelami' a Genova nel Medioevo vedi U. Formentini, L'arte romanica genovese ed i "magistri antelami”, in Storia di Genova dalle origini al tempo nostro, vol. 3: Il Comune dei Consoli, ed. A. Regolo Scarsella Milano, 1942, pp. 275-31; C. Dufour Bozzo, La porta urbana del Medioevo: Porta Soprana di Sant'Andrea in Genova: immagine di una città, Roma, 1989, in part. pp. 213-229; A. Dagnino, Gli scultori nei cantieri dei magistri antelami, in Niveo de marmore, op. cit. (n. 2), pp. 131-133; A. Decri, La presenza degli Antelami nei documenti genovesi, in Magistri d'Europa, op. cit. (n. 12),
} 
maso di Fassolo, e le porte di Sant'Andrea e di Santa Fede ${ }^{14}$. Infatti, alcuni studiosi ipotizzano una sorta di monopolio dei magistri antelami nei cantieri genovesi del periodo romanico ${ }^{15}$, mentre altri ricostruiscono una collaborazione con altre maestranze lombarde, ad esempio quelle di Campione ${ }^{16}$. La problematica dipende anche dalle divergenze di stile e tipoligia delle opere di scultura architettonica sulle quali ci si interroga se possano essere attribuite ai magistri antelami.

Vista la scarsità dei documenti in proposito, è impossibile fare chiarezza nei singoli casi. Un monopolio assoluto pare improbabile, ma d'altro canto non è imverosimile che, anche se non si trattava di monopolio, i magistri antelami abbiano avuto un ruolo considerevole nei lavori di costruzione e di scultura architettonica a Genova nel XII secolo; come approfondirò in altra sede, è possibile ricorrere di certe tipologie e di sigle formali nelle loro sculture architettoniche.

A proposito del fatto che le maestranze antelamiche esercitavano la loro professione sia a Genova che a Fidenza, credo meriti concentrare l'attenzione sulla famiglia Pallavicino, di ceppo Obertenghi, feudatari fedelissimi all'impero, che dominavano sulla parte occidentale

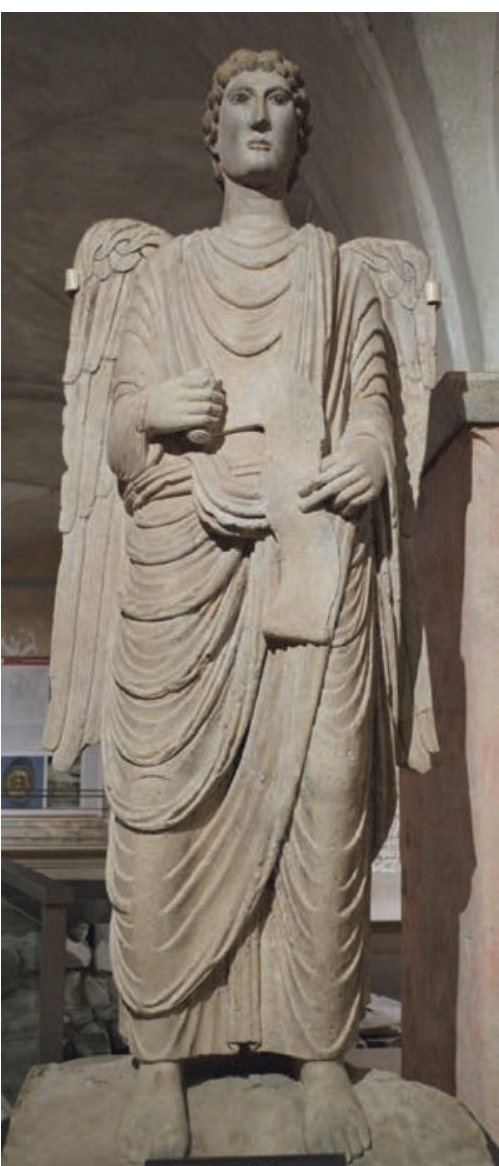

Fig. 17. Museo diocesano di Parma, Angelo dal Battistero di Parma

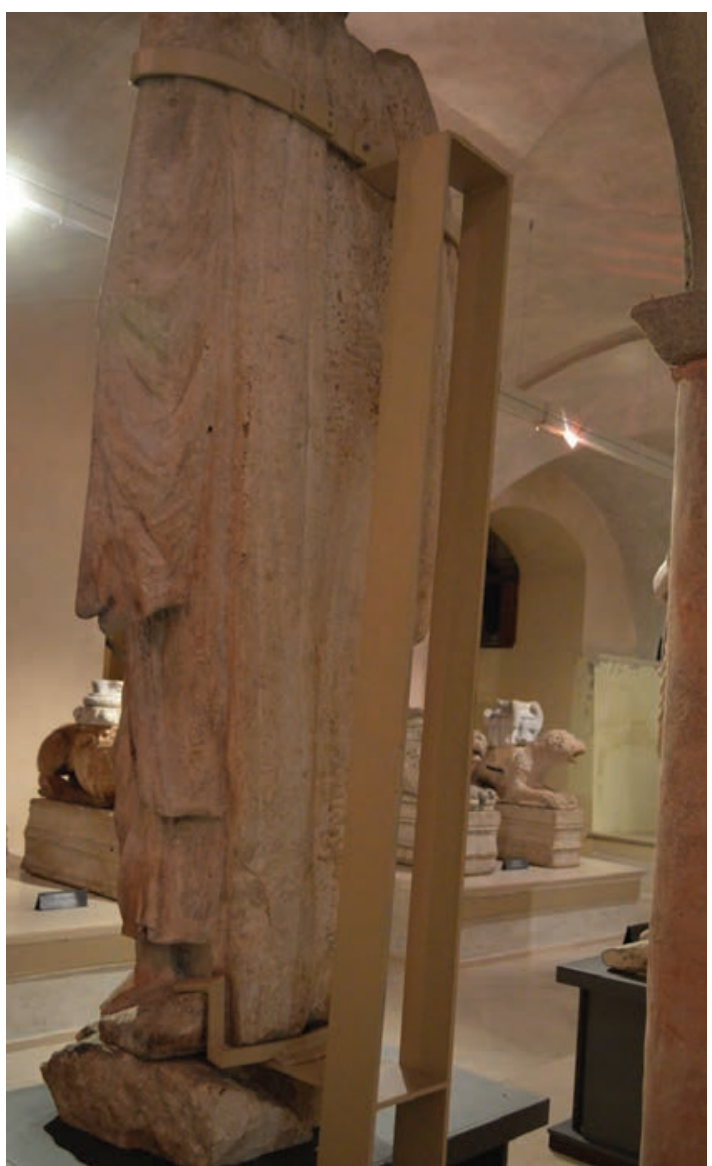

Fig. 18. Museo diocesano di Parma, Angelo dal Battistero di Parma, retro
dell'Emilia e specialmente su Borgo San Donnino, località che faceva in pratica parte del cosiddetto 'Stato Pallavicino'. L'attuale Duomo di Fidenza venne eretto anche grazie al forte sostegno dei Pallavicino ${ }^{17}$.

È da notare che a Genova da circa la metà del XII secolo esisteva un ramo importante della famiglia Pallavicino. I Pallavicino di Genova ebbero come capostipite Niccolò, nipote di Oberto Pallavicino, menzionato per la prima volta con l'appellativo di Pelavicinus ed attivo nella prima metà del XII secolo nell'area di Fidenza e Busseto. Alberto, padre di Nic- colò e figlio di Oberto partecipò alle crociate con i Genovesi. Suo figlio Niccolò, capostipite dei Pallavicino genovesi, è documentato per la prima volta a Genova nel 1154. Il figlio di questo Niccolò, Giovanni, sposò Maria Fieschi, e nel 1225 venne eletto consigliere comunale. La famiglia assunse notevole prestigio ed importanza nella città di Genova. Come scrive Gabriella Airaldi, storico genovese, nel suo libro sulla Genova del Medioevo, circa nella metà del XII secolo tra le famiglie più potenti della città si annoverano insieme alle casate celebri dei Fieschi, Embriaco e Doria, anche i Pallavicino ${ }^{18}$. Date le cir-

pp. 407-432; F. Zoni, Magistri antelami tra Genova, Liguria di ponente e Ventimiglia: attestazioni documentarie e alcune considerazioni (secoli XII - XIII), in Intemelion 19, 2013, pp. 5-22; Idem, Le maestranze antelamiche nella Liguria di ponente, diffusione dell'”Opus quadratum" tra XII e XIII secolo, in Archeologia dell'architettura 18, 2014, pp. 229-244.

${ }_{14}$ E. Poleggi, Santa Maria di Castello e il Romanico a Genova, Genova, 1973; C. Dufour Bozzo, Il reimpiego dei marmi antichi nei monumenti medievali e l'esordio della scultura architettonica del "Protoromanico" a Genova, in Bollettino d'arte S.6, 64, 1979, pp. 1-58; C. Di Fabio, Ricerche di architettura altomedievale e romanica a Genova: il monastero di San Tommaso, in Storia monastica ligure e pavese: studi e documenti, Cesena, 1982, pp. 103-171; C. Dufour Bozzo, La porta urbana, op. cit. (n. 13), in part. 213-229; C. Di Fabio, Scultura e allegoria politica nella Genova romanica: i capitelli della Porta di Santa Fede, in Immagini del Medioevo: Studi di arte medievale per Colette Dufour Bozzo, ed. A. Dagnino et. al., Genova, 2013, pp. 97-106.

${ }^{15}$ In part. E. Poleggi, Santa Maria di Castello, op. cit. (n. 14); Idem, Città e magstri antelami, una storia seguestrata, in Magistri d'Europa, op. cit. (n. 12), pp. 389-406.

${ }^{16}$ S. Lomartire, Comacini, Campionesi, Antelami, “Lombardi”, op. cit. (n. 12).

${ }^{17}$ Emilio Nasalli Rocca, storico piacentino, ha pubblicato diversi studi, tuttora validi, sui Pallavicino in Emilia occidentale: Idem, La lapide tombale del Marchese Guido Pallavicino a Fontevivo (1301). Contributo agli studi sull'Ordine Cistercense e sull'Ordine dei Templari, in Archivio storico per le provincie parmensi S.4, 4, 1954, pp. 79-86; Idem, La Signoria di Oberto Pallavicino nelle formulazioni dei suoi atti di governo, in Archivio storico lombardo 83, 1956, pp. 29-43; Idem, Vescovi, città, signori sui confini tra Parma, Piacenza, Cremona, in Archivio storico lombardo 91-92, 1966, pp. 135-161; Idem, La posizione politica dei Pallavicini dall'età dei Comuni a quella delle Signorie, in Archivio storico per le provincie parmensi S.4, 20, 1968, pp. 65-113. Sui Pallavicino i volumi di Pompeo Litta sono sempre fontamentali: Idem, Famiglie celebri litaliane, S.1: Pallavicino, tav. I (1838), tav. XIV (1840), Firenze. Vedi inotre, E. Seletti, La città di Busseto, capitale un tempo dello Stato Pallavicino, Milano, 1883; C. Soliani, Nelle terre dei Pallavicino, vol.I-1, Storia civile e politica dell'antico, Oltre Po cremonese dalle origini alla fine del XV secolo, Parma, 1989; U. P. Censi, Nelle terre dei Pallavicino, vol.II, Economia, società e cultura, Parma, 1990. Cf. anche M. P. Branchi, La committenza obertenga lungo la Via Francigena, in Medioevo: la chiesa e il palazzo, atti del convegno (Parma, 20-24 settembre 2005) ed. A. C. Quintavalle, Milano, 2007, pp. 448-455.

${ }^{18}$ G. Airaldi, Genova e Liguria nel medioevo, Torino, 1986, p. 110. 


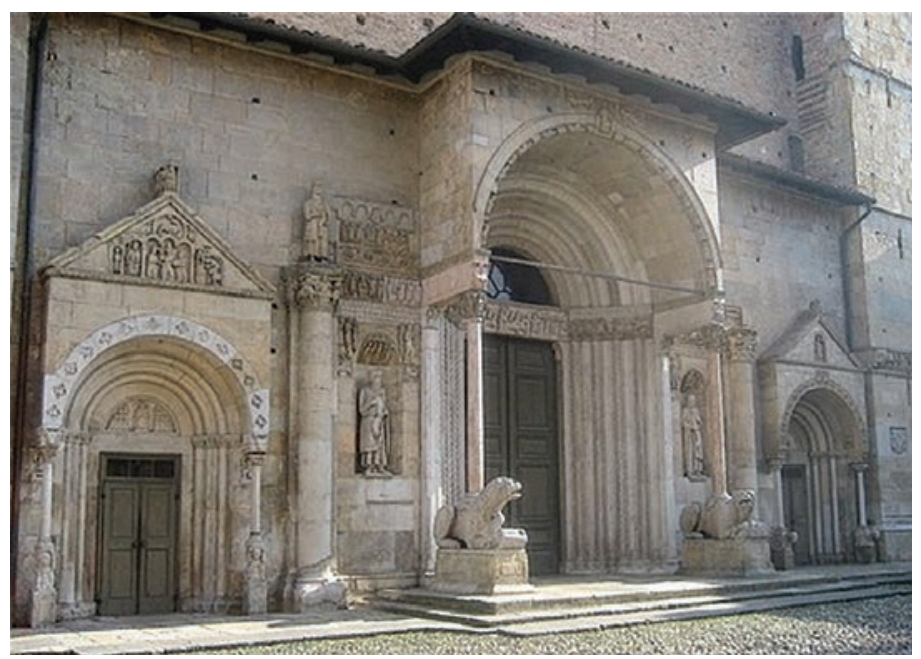

Fig. 19. Cattedrale di Fidenza, facciata

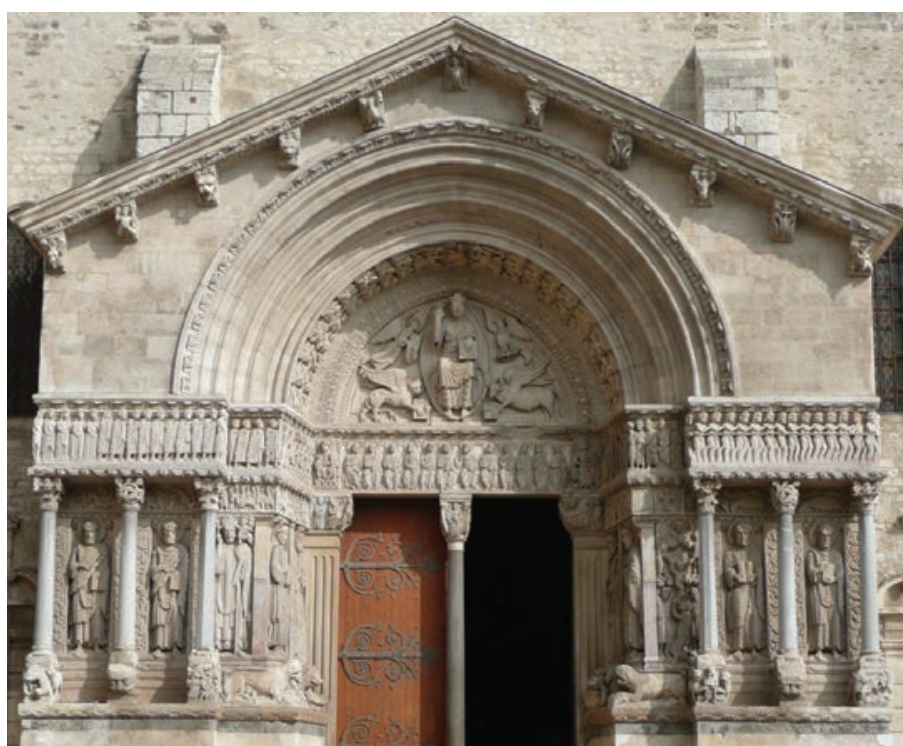

Fig. 21. Arles, Saint-Trophime, facciata

costanze, sembra probabile che i Pallavicino abbiano avuto un ruolo non trascrabile negli spostamenti delle maestranze antelamiche tra Liguria e Emilia. Anche l'esistenza della Via Francigena, che collegava il Nord-Est e il Sud-Ovest degli Appennini ${ }^{19}$, deve avere avuto una certa importanza, così come le cave di Carrara.

Dall'altro canto non si deve dimenticare il ruolo del porto di Genova, città che proprio a partire da quell'epoca era una delle più potenti 'repubbliche marinare'. Nel XII e nel XIII secolo Genova aveva forti rapporti mercantili coll'area provenzale e linguadocana, lungo il litorale fra Marsiglia

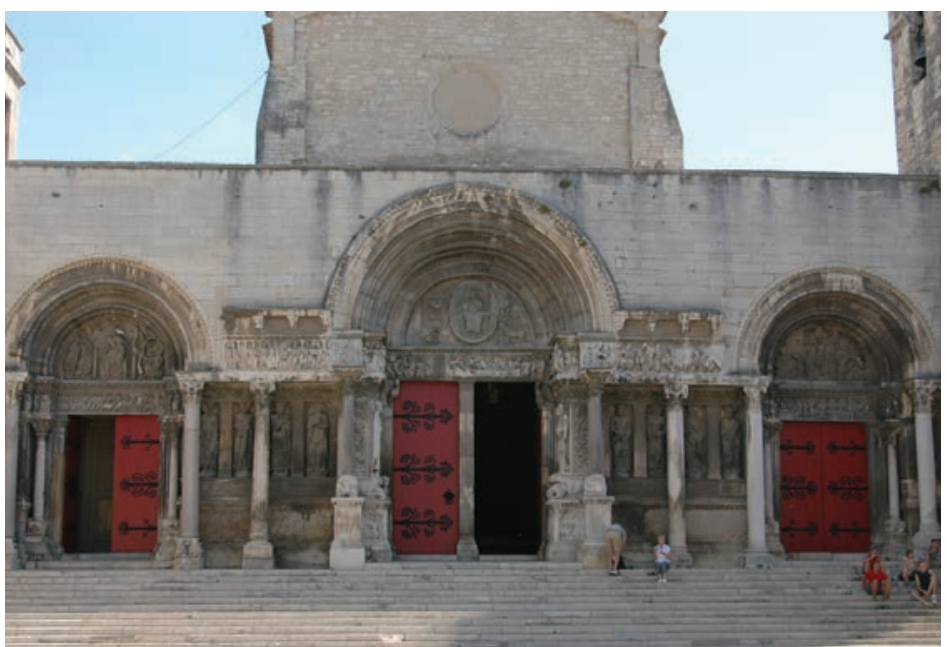

Fig. 20. Saint-Gilles-du-Gard, facciata

e Montpellier ${ }^{20}$. Tra i centri di questa area, Aigues Mortes divenne nel XIII secolo di fatto proprietà di Guglielmo Boccanegra di Genova ${ }^{21}$. Adiacente a Aigues Mortes si trovava il monastero di Psalmodi, di origine carolingia, che nei decenni tra il 1170 e il 1200 circa divenne molto più grande e importante. Significativo è che l'abbazia di Saint-Gilles du Gard, Arles, Psalmodi e Aigues Mortes sono località ubicate a poca distanza l'una dall'altra.

Queste circostanze sociali e politiche dell'epoca farebbero pensare che le maestranze antelamiche abbiano avuto contatto con l'arte provenzale proprio tramite Genova. Per quanto riguarda il monastero di Psalmodi, decaduto e distrutto in età moderna, grazie alla campagna di scavi, condotti tra 1970 e 1988, sono stati rintracciati resti della costruzione della fine del XII secolo, caratterizzata da elementi gotici, come le volte a sesto acuto costolonate, e contemporaneamente anche da un richiamo all'arte antica, in particolare nei motovi decorativi $^{22}$. Queste sono caratteristiche non solo dell'architettura locale, come Saint-Gilles-du-Gard e Saint-Trophime, ma di monumenti riferiti dalla critica a Benedetto Antelami o alle maestranze antelamiche a Genova, Fidenza e Parma ${ }^{23}$. Daltronde, visto lo stato attuale pesantemente danneggiato del complesso monastico di Psalmodi, è assai difficoltoso approfondire questo argomento.

Riguardo agli interscambi artistici tra Provenza e Liguria dal primo periodo romanico sino a circa la metà del XII secolo, Fulvio Cervini ha espresso un'opinione piuttosto negativa ${ }^{24}$. Invece certe tipologie architettoniche delle chiese e degli ospedali dei Cistercensi e degli ordini cavallereschi dalla metà del XII secolo in Liguria e nella stessa Genova tesmoniano di rapporti artisitici con la Francia inclusa la

${ }^{19}$ F. Opll, L'attenzione del potere per un grande transito sovraregionale: il Monte Bardone nel XII sec., in Quaderni storici 21, 1986, pp. 57-75.

${ }^{20} \mathrm{Cf}$. H. G. Krueger, Navi e proprietà navale a Genova, seconda metà del sec. XII, Genova,1985; Idem, The Genoese travelling merchant in the twelfth century, in Journal of European Economic History 22, 1993, pp. 251-283; Quentin Van Doosselaere, Commercial agreements and social dynamics in Medieval Genoa, New York, 2009.

${ }^{21}$ B. Sournia, Les fortifications d'Aigues-Mortes, in Congrès Archéologique de France 134, 1979, pp. 9-26.

${ }^{22}$ Sull'abbazia di Psalmodi vedi Jerrilyn Dodds, L'ancienne abbaye de Psalmodi (Saint-Laurent-d'Aigouze, Gard) premier bilan des fouilles (1970-1988), in Archéologie médiévale 19, 1989, pp. 7-57.

${ }^{23}$ G. de Francovich, Benedetto Antelami, op. cit. (n. 1); R. Wagner-Rieger, Die italienischen Baukunst zu Beginn der Gotik, vol. 1, Oberitalien, Graz-Köln, 1956; J. Thirion, Saint-Trophime d'Arles, in Congrès Archéologique de France 134, 1979, pp. 360-479; S. Lomartire, Introduzione all'architettura del Battistero di Parma, in Benedetto Antelami e il Battistero di Parma, op. cit. (n. 1), pp. 145-25o; Y. Kojima, Storia di una cattedrale, op. cit. (n. 1); A. Hartmann-Virnich et al., Saint-Gilles-du-Gard: nouvelles recherches sur un monument majeur de l'art roman, in Bulletin monumental 171-4 (numero supeciale), 2013; Idem, La cathédrale Saint-Trophime d'Arles: réflexions sur les antécédents de l'église romane et de son espace claustral, in Les Cahiers de Saint-Michel de Cuxa 44, 2013, pp. 55-73,

${ }^{24}$ F. Cervini, Tra Liguria e Provenza al tempo del "premier art roman": mito e realtà di uno snodo internazionale, in Intemelion 20, 2014, pp. 23-42. 


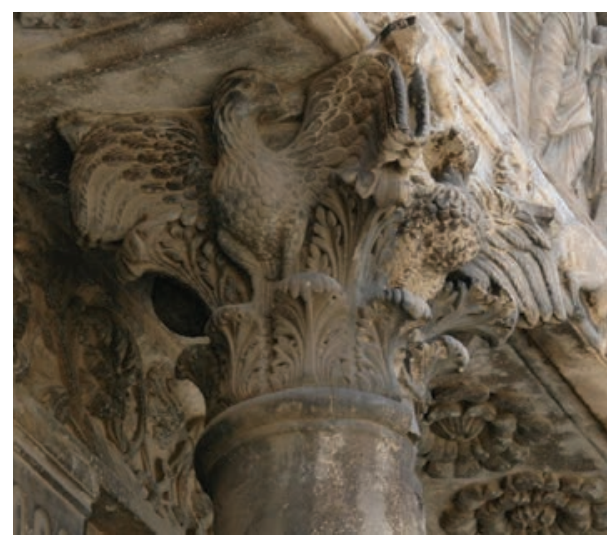

Fig. 22. Saint-Gilles-du-Gard, facciata, capitello con le Aquile

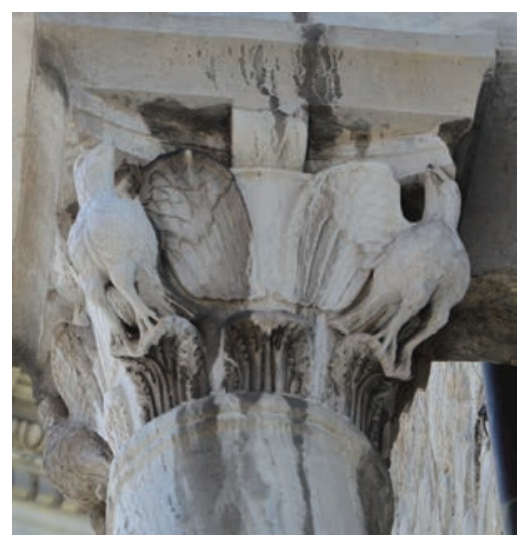

Fig. 23. Genova, Porta Soprana o Sant'Andrea, interno, capitello con le Aquile,

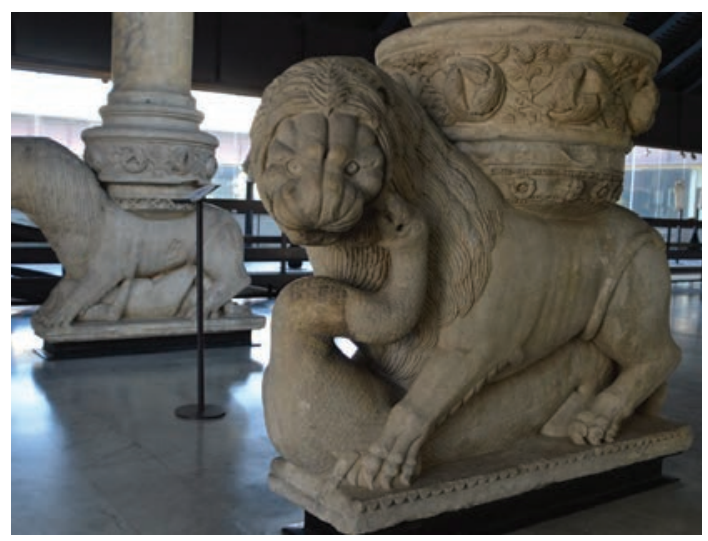

Fig. 24. Genova, Museo di Sant'Agostino, Leoni stilofori, dalla chiesa di San Siro di Genova

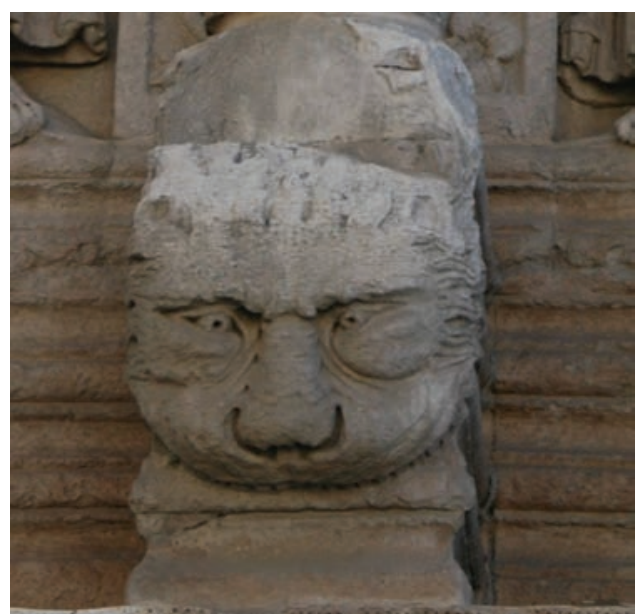

Fig. 25. Arles, Saint-Trophime, facciata

Provenza, come del resto sostengono Colette Dufour Bozzo e lo stesso Cervini ${ }^{25}$. È osservazione significativa, in quanto, come discuterò in altra sede, sembra che quegli ordini fossero legati agli influssi dell'architetura e scultura architettonica francese sui cantieri 'antelamici'. D'altronde, Clario di Fabio ha individuate una penetrazione del liguaggio scultoreo del primo Gotico francese nell'arte genovese della seconda metà del XII secolo nell'Angelo simbolo di san Matteo evangelista proveniente dalla chiesa di Santa Maria delle Vigne a Genova e la Madonna col Bambino, ora nel convento dei frati Cappuccini di Santa Margherita Ligure ${ }^{26}$.

In questo quadro artistico, non sarebbe inopportuno prendere in considerazione anche le Aquile, di carattere alquanto classicheggiante della Porta Soprana o di Sant'Andrea a Genova del 1155, che sembrano costituire una variazione semplificata e stilizzata di quelle della facciata della

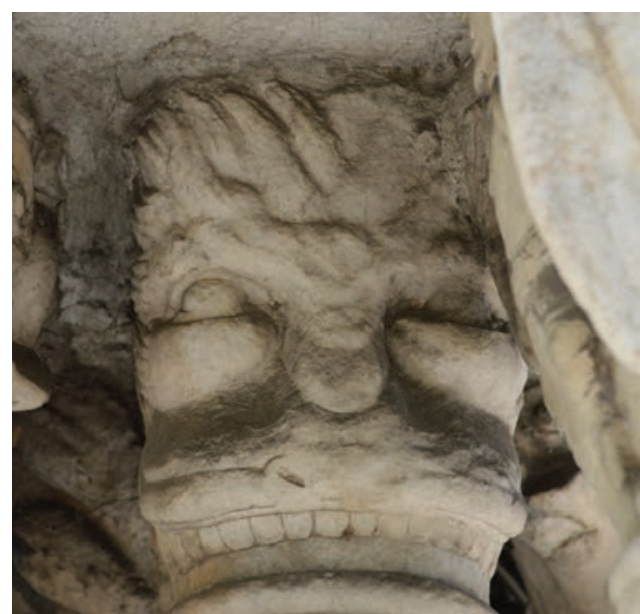

Fig. 26. Genova, Chiostro di Sant'Andea della Porta, part. di un capitello

chiesa di Saint-Gilles-du-Gard (figg. 22, 23) ${ }^{27}$. Inoltre, sono elementi comuni anche i girali con fogliame che si avolge a spirale della base dei leoni stilofori del Museo di Sant'Agostino provenienti dalla chiesa di San Siro (figg. 14, 24) e le caratteristiche protomi di leoni che divorano le colonne nel chiostro di Sant'Andrea della Porta (figg. 25-26) ${ }^{28}$. Queste ultime si ritrovano anche nel Duomo di Fidenza e nel battistero di Parma (ff. 27-30). Ma vorrei rimandare ad altra occasione l'analisi dei rapporti stilistici ed anche potilico-religiosi tra la Liguria e le città dell'Emila occidentale, rapporti che riguardano anche l'ordine cistercense. Invece, qui, mi limito a sottoporre a revisione la cronologia delle sculture antelamiche a Fidenza in rapporto ai cantieri della Provenza.

Per quanto riguarda la datazione dei Profeti nella facciata del Duomo di Fidenza, ritengo che siano circa della fine del terzo quarto del XII secolo, come si vedrà qui di seguito ${ }^{29}$.

${ }_{25}$ C. Dufour Bozzo, Culture architettoniche a confronto: spunti inediti per i rapporti fra la Liguria occidentale e Genova con la Provenza: S. Maria in Latronorio, S. Pietro in Vesima, S. Donato in Genova, in Rivista ingauna e intemelia N.S, 51, 1996, pp. 103-106; F. Cervini, Architettura gerosolimitane nel ponente ligure, in Idem, La pietra e la croce, Ventimglia, 2005, pp. 98-125.

${ }^{26}$ C. Di Fabio, La "Madonna" di S. Margherita e il "David" di Pisa, due esperienze nella scultura europea alla fine del XII secolo, in Storia dell'arte 44/46, 1982, pp. 35-44; Idem, Scultura a Genova 1160-1259: la ricezione del gotico: inediti e spunti di ricerca, in Bollettino d'arte S.6, 71, 1986, 39/40, pp. 143-160; Idem, Geografia e forme della scultura in Liguria, in La scultura a Genova e in Liguria, vol. 1: Dalle origini al Cinquecento, Genova, 1987, pp. 87-129; Idem, Scultore francese (1180-119o). Maria Regina, in Imago Mariae: tesori d'arte della civiltà cristiana, catalogo della mostra (Roma, 20 giugno-2 ottobre 1988), Milano, 1988, pp. 75-76; Idem, scheda 30: Concio d'arcata con Angelo, simbolo di san Matteo evangelista, scheda 31: Madonna col Bambino, in Niveo de Marmore, op. cit. (n. 2), pp. 156-158.

${ }^{27}$ Sulle Aquile della Porta di Sant'Andrea Dufour Bozzo sostiene che agli antelami è forse legittimo fare riferimento soltanto per capitello dell'aquila che pare di fattura più sommaria e modesta. C. Dufour Bozzo, La porta urbana, op. cit. (n. 13), p. 225.

${ }^{28}$ Riguardo al motivo a girali delle basi delle colonne dei leoni stilofori da San Siro, Di Fabio già indicava: «forse sulla via della Provenza». C. Di Fabio, Geografie e forme della scultura in Liguria, op. cit. (n. 26), p. 90.

${ }_{29}$ Cf. Y. Kojima, Storia di una cattedrale, op. cit. (n. 1), pp. 61-87. 


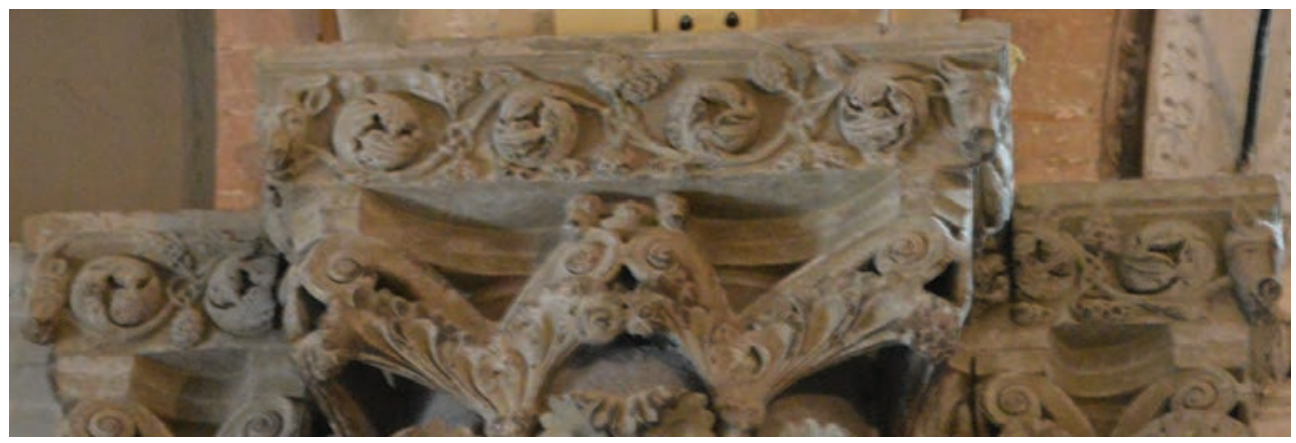

Fig. 27. Cattedrale di Fidenza, capitelli della navata meridionale

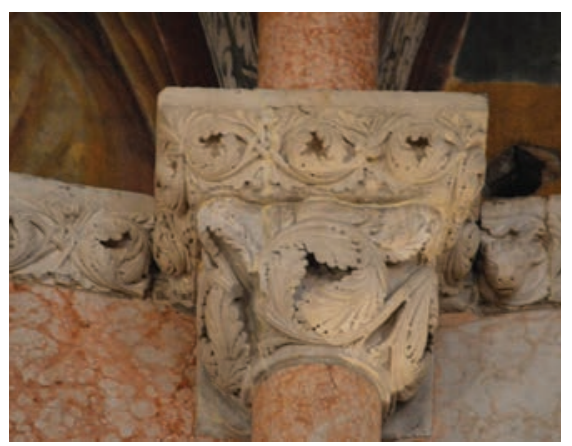

Fig. 28. Battistero di Parma, interno, capitello allimposta della cupola

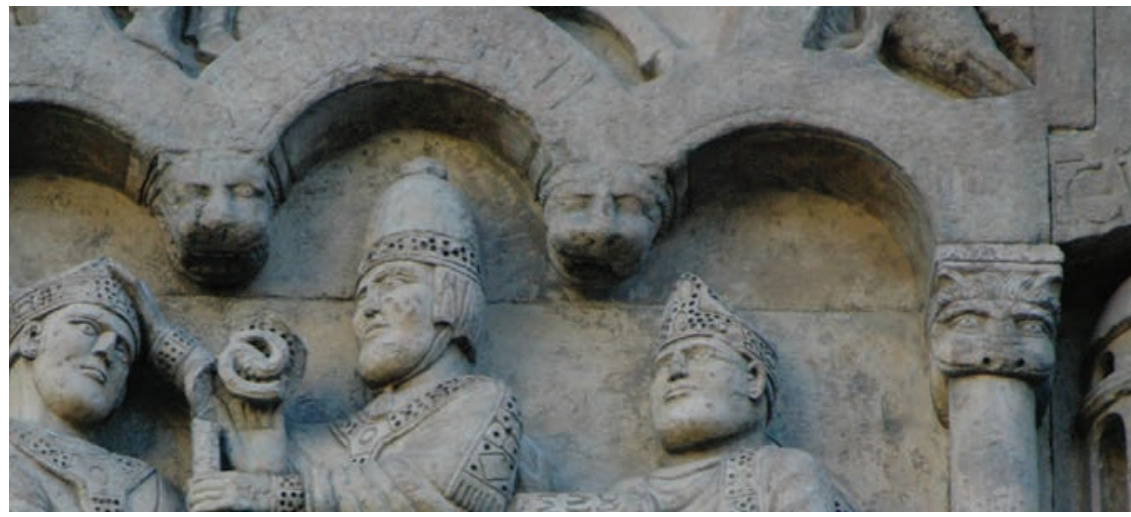

Fig. 29. Cattedrale di Fidenza, facciata, part. del protiro settentrionale

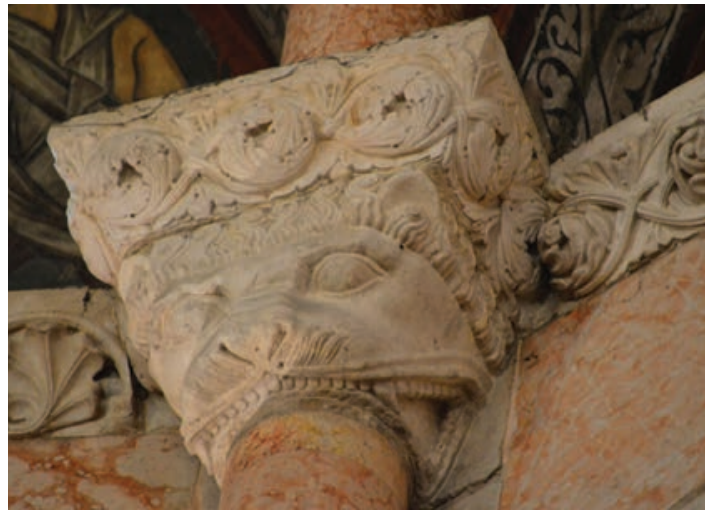

Fig. 30. Battistero di Parma, interno, capitello all'imposta della cupola
La costruzione della chiesa attuale venne avviata dopo la distruzione della città di Borgo San Donnino da parte dei Parmensi nel 1152. Ebbe un peso rilevante nell'avvio della fabbrica un privilegio concesso nel 1162 da Federico Barbarossa a Guglielmo Pallavicino, che faceva parte della corte dell'imperatore. Sappiamo che nel 1179 il corpo di san Donnino, patrono della città, era già provvisoriamente conservato in un monastero gentilizio dei Pallavicino a causa dei lavori nella cripta, che venne consacrata nel 1207, nell'anno in cui il corpo di san Donnino ritornò in questo ambiente. Ė chiara la divergenza stilistica tra il linguaggio protogotico della cripta, specie nei capitelli a crochet, e quello romanico della parte inferiore della facciata e delle navate (figg. 31. 32). Queste parti 'romaniche' dell'edificio quindi sembrano essere state ultimate entro il 1179 circa. Capitelli cubici quasi identici a quelli delle navate del Duomo di Fidenza, a cubo scantonato con profirature e nervature angolari, sono presenti nei resti del Palazzo Imperiale del Barbarossa o dell'Arena a Parma (figg. 33-35) ${ }^{30}$. Gli studiosi concordano sul fatto che il palazzo sia già stato ultimato nel 1162. Nelle navate del Duomo di Fidenza, oltre ai capitelli cubici ora visti ne esistono anche di tipo corinzio, di una resa formale pressoché identica a quella della semicolonna a destra e del protiro meridionale nella facciata (figg. 36, 37). Le foglie appuntite carnose e dense si arricciano e si piegano mollemente. Pertanto, dal momento che l'architettura e la scultura della facciata costituiscono un insieme organico earmonico, i Profeti devono essere stati progettati nel momento iniziale dei lavori della facciata (fig. 19).

Dalle osservazioni fatte finora, non sembra troppo azzardato collocare i Profeti circa alla fine del terzo quarto del

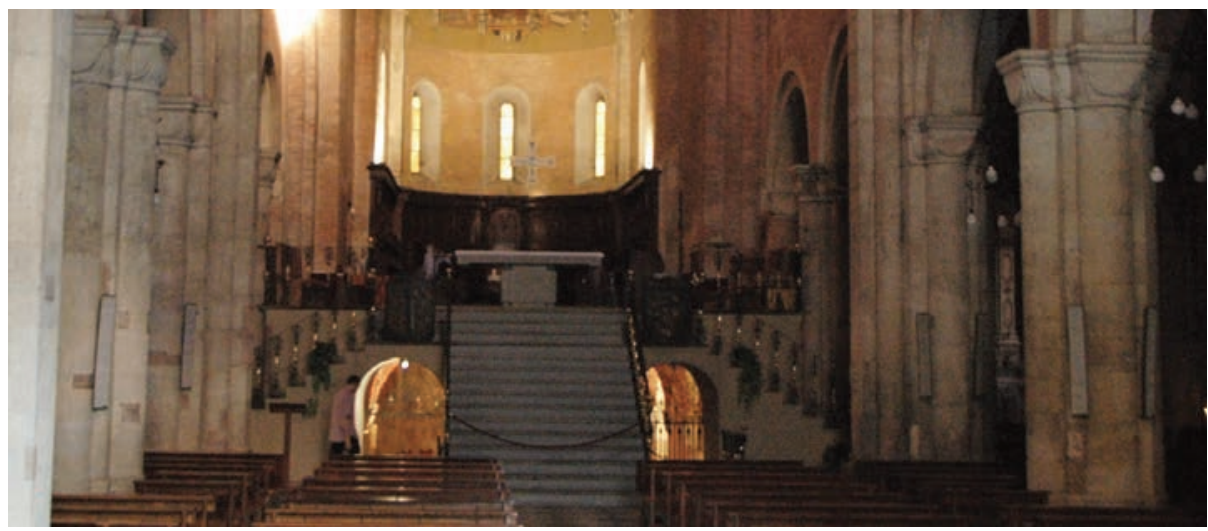

Fig. 31. Cattedrale di Fidenza, parte inferiore delle navate

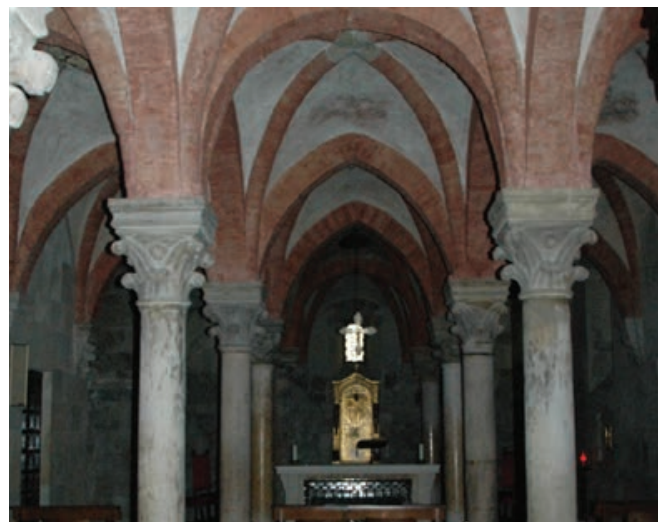

Fig. 32. Cattedrale di Fidenza, cripta

\footnotetext{
30 Sul Palazzo Imperiale o dell'Arena di Parma vedi C. Guzzon, Il Palazzo dell'Arena. Uno dei più significativi edifici di Parma attraverso ricostruzioni e rifacimenti durati quasi duemila anni, in Archivio storico per le province parmensi S.6, 47, 1995, pp. 247-261; G. Valenzano, Sulle tracce del Palazzo Imperiale, in Federico II e L'Emilia Occidentale, catalogo della mostra (San Secondo e Parma, 8 aprile—28 maggio 1995), Parma, 1995, pp. 35-44.
} 


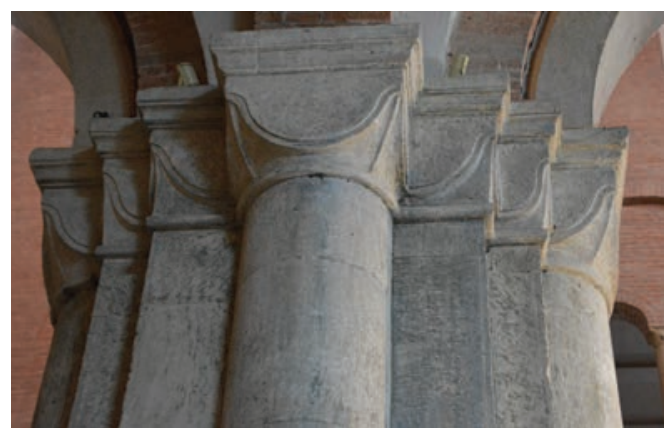

Fig. 33. Cattedrale di Fidneza, capitelli delle navate

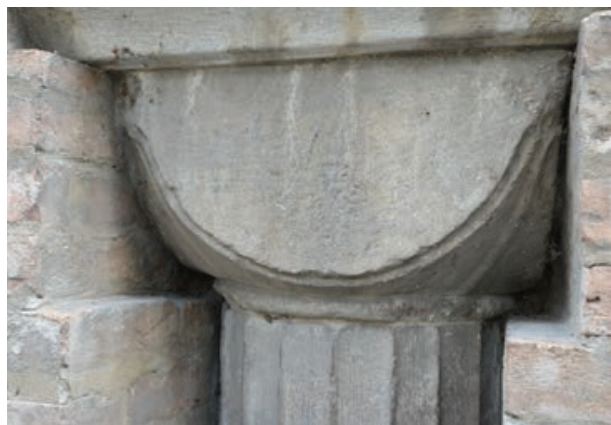

Fig. 34. Parma, resti del Palazzo Imperiale del Barbarossa o d'Arena

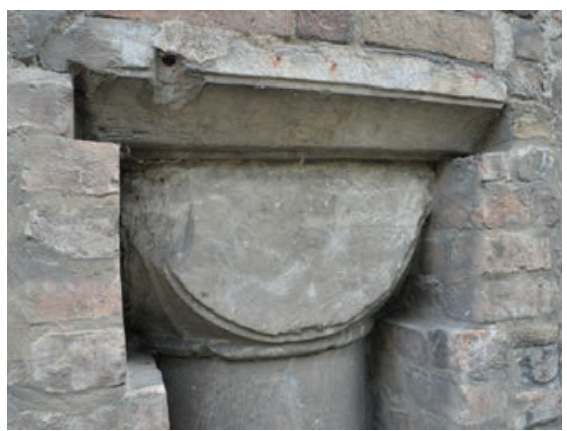

Fig. 35. Parma, resti del Palazzo Imperiale del Barbarossa o d'Arena

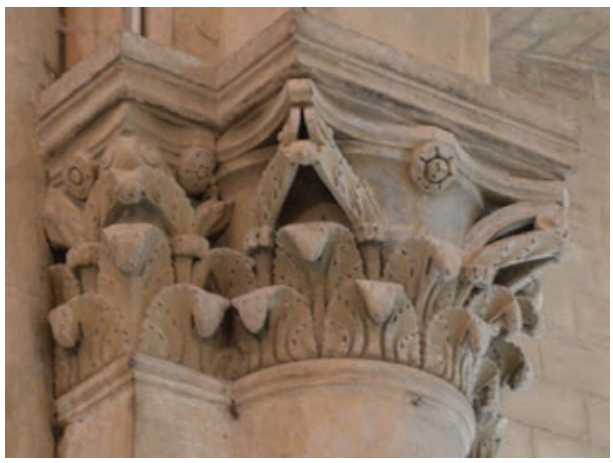

Fig. 36. Cattedrale di Fidenza, capitello della navata settentrionale

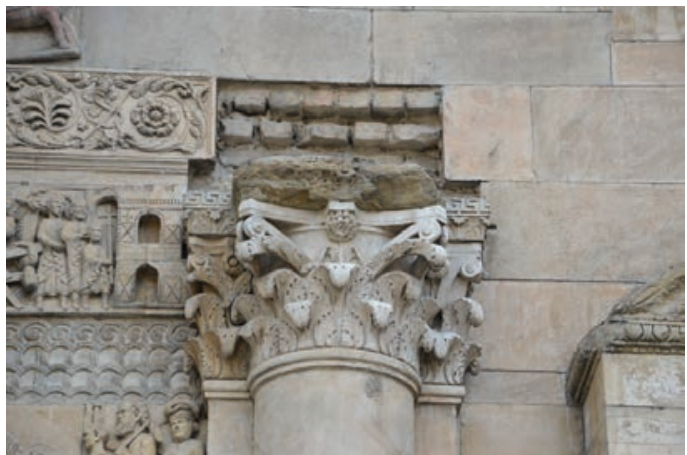

Fig. 37. Cattedrale di Fidenza, facciata, capitello della semicolonna meridionale

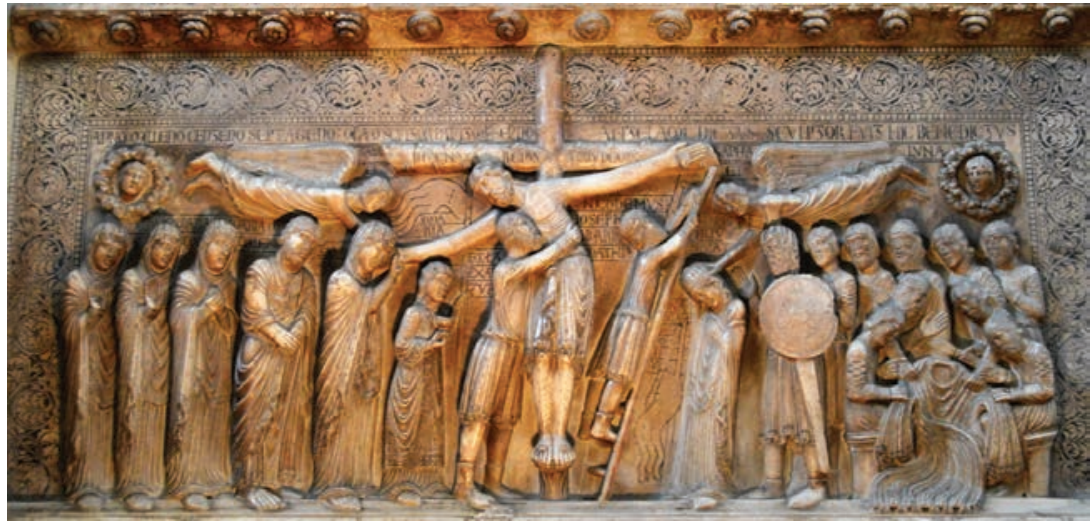

Fig. 38. Cattedrale di Parma, Benedetto Antelami, Deposizione

XII secolo, e quindi forse prima della celebre Deposizione di Benedetto Antelami nel Duomo di Parma del 1178. Come è noto nell'iscrizione sulla lastra della Deposizione si legge «ANNO MILLENO CENTENO SEPTVAGENO : OCTAVO SCVLTOR PAT(ra)VIT M(en)SE SECV(n)DO / ANTELAMI DICTVS SCVLPTOR FVIT HIC BENEDICTVS» (fig. 38). Perciò l'Antelami nel 1178 non «PATVIT (si rivelò)» ma «PATRAVIT (esegui)», come letto giustamente sin dal Ottocento da Max Georg Zimmermann, e successivamente da Adolfo Venturi ${ }^{3}$.
A proposito del rapporto cronologico con le sculture provenzali, di recente Andreas Hartmann-Virnich, basandosi su analisi archeologiche molto minuziose condotte in occasione dei lavori di restauro di questi monumenti, ha proposto una datazione per la decorazione plastica della facciata di Saint-Gilles-du-Gard al terzo quarto del XII secolo, e per il complesso di Saint-Trophime di Arles, tra 1185 e 1200 circa per l'ala nord del chiostro, all'ultimo decennio del XII secolo per il portale, e tra 1200 e 1220 circa per l'ala est del chiostro ${ }^{32}$.

\footnotetext{
${ }^{31}$ M. G. Zimmermann, Oberitalische Plastik, op. cit. (n. 5), pp. 104-106; A. Venturi, Storia dell'arte italiana, vol.III, Milano, 1904, p. 340. Chi per primo ha letto «PATVIT» è stato Kinsgley Porter, il quale, come obiettato da Bognetti, avrebbe male interpretato un tratto sopra la «T». Come sappiamo, questo tipo di tratto sopra una o più lettere indica sempre un'abbreviazione. Arthur Kingsley Porter, Lombard Architecture, 3 voll. di testo e 1 vol. di tavole, New Haven, 1917, vol. 3, p. 161; G. P. Bognetti, Una rettifica epigrafica, a proposito dei limiti cronologici dell' opera dell' Antelami, in Bayerische Akademie der Wissenschaften, Philosophisch-Historische Klasse, Sitzungsberichte 3, 1959, pp. 3-11.

${ }^{32}$ A. Hartmann-Virnich, La mise en oeuvre d'un chef d'oeuvre roman: le portail de Saint-Trophime d'Arles, in Archeologia 314, 1995, pp. 68-73; Idem (ed.), Le portail de Saint-Trophime d Arles. Naissance et renaissance d'un chef- d'œeuvre roman, Arles, 1999; Idem (con la collaborazione di H. Hansen), La façade de l'abbatiale de Saint-Gilles-du-Gard, in Congrès archéologique de France 157, 2000, pp. 271-291; Idem, Les galeries romanes du cloitre de Saint-Trophime d'Arles: études sur un chantier de prestige, in Der mittelalterliche Kreuzgang: Bautyp, Ausstattung, Funktion und Symbolik, atti del convegno (Tübingen,10-12 giugno1999), ed. Peter Klein, Regensburg, 2004, pp. 285-316; Idem, L'inscription de la fondation de l'abbatiale de Saint-Gilles-du-Gard: réflexions sur un
} 
Per quanto riguarda il chiostro di Saint-Trophime di Arles rimangono aperte alcune questioni, in particolare la datazione delle sculture delle gallerie nord ed est del chiostro. Nel piedistallo dell'altorilievo di San Trofimo nella galleria nord si trova l'epitaffio del prevosto Jourdain con la data di morte 1188 , anno che è stato considerato terminus post quem per questa parte del chiostro, ma non è da escludere la possibilità di un inserimento posteriore, come Whitney Stoddard aveva già sosostenuto nel $1973^{33}$. Secondo me le sculture delle due gallerie romaniche del chiostro sarebbero anteriori stilisticamente a quelle del portale di Saint-Trophime, e l'intero complesso arlesiano potrebbe essere contempraneo o di poco successivo alla facciata di Saint-Gilles. Per quanto le datazioni dei due cantieri di Saint-Gilles e Arles siano molto incerte, ritengo probabile che vadano collocate più o meno negli stessi decenni dell'attività di Benedetto Antelami e delle maestranze antelamiche di cui faceva parte a Fidenza, forse con lieve anteriorità della Provenza sull'Emilia.

Pertanto sembra plausibile che il rapporto tra il linguaggio della scultura monumentale attestato a Fidenza e quello che troviamo negli stessi decenni in Provenza, oltre che per la comune eredità dell'arte antica possa essere spiegato non solo tramite i viaggi degli artisti, ma in relazione ai contatti storici e politici, soprattutto quelli inerenti al porto di Genova, alla famiglia Pallavicino e ai Cistercensi. In questo contesco sociale, nelle quali i modelli artistici e gli artisti stessi si potevano incontrare, circolava una koinè caratterizzata da una parte dal forte classicismo, come attestano le sculture provezali e fidentine, dall'altra dalla apertura al nuovo lessico protogotico.

problème archéologique, in Le plaisir de l'art du Moyen Âge, mélanges en hommage à Xavier Barral i Altet, ed. R. Alcoy et al., 2012, pp. 140-148; A. HartmannVirnich et al., Saint-Gilles-du-Gard: nouvelles recherches, op. cit. (n. 23); A. Hartmann-Virnich, La cathédrale Saint-Trophime d’Arles: réflexions, op. cit. (n. 23); H. Hansen, A. Hartmann-Virnich, La façade de l'abbatiale de Saint-Gilles-du-Gard: nouvelles recherches sur la construction d'un chef d'oeuvre de l'art roman, in Les Cahiers de Saint-Michel de Cuxa 45, 2014, pp. 157-173. Vedi anche W. S. Stoddard, The Façade of Saint-Gilles-du-Gard: its influence on French sculpture, Middletown (Conn.), 1973; J. Lacoste, La galerie nord du cloître de Saint-Trophime d'Arles, in Les Cahiers de Saint-Michel de Cuxa 7, 1976, pp. 127-162; D. Diemer, Untersuchungen zu Architektur und Skulptur der Abteikirche von Saint-Gilles, Stuttgart, 1978; J. Thirion, Saint-Trophime d'Arles, op. cit. (n. 23); J. F. Scott, St.-Gilles-Du-Gard: the west facade figured frieze: irregularities and relative chronology, Frankfurt am Main, 1981.

33 W. S. Stoddard, The Façade of Saint-Gilles-du-Gard, op. cit. (n. 32), p. 259. 